\title{
Metodología de evaluación mediante la realización de pruebas de evaluación activa circular tipo test
}

N. Montañés, O. Fenollar, V. Fombuena, R. Balart, D. García-Sanoguera

Universitat Politècnica de València. Departamento de Ingeniería Mecánica y de Materiales. Plaza Ferrándiz y Carbonell, 03801 Alcoy (Alicante) España. e-mail: ocfegi@epsa.upv.es

\begin{abstract}
In recent years the concept of evaluation has reached a significant transformation. The traditional concept of evaluation as a final act, has been replaced by a concept of evaluation as something intrinsic to the learning process, creating interactive and circular relationships between learning and evaluation. In this sense, we have introduced a part of the act of assessment in an intermediate stage of learning, so that will be producing a feedback between the results obtained and the lessons learned.
\end{abstract}

Keywords: learning, skills, training, methodology, evaluation .

\begin{abstract}
Resumen
En los últimos años el concepto de evaluación ha sufrido una importante transformación. De la tradicional concepción de evaluación como acto final, hemos pasado a un concepto en el que incluimos la evaluación como algo intrínseco al proceso de aprendizaje, creándose relaciones interactivas y circulares entre aprendizaje y evaluación. En este sentido, hemos introducido una parte del acto de evaluación en una etapa intermedia del aprendizaje, de tal forma que se vaya produciendo una retroalimentación entre los resultados obtenidos y el aprendizaje adquirido.
\end{abstract}

Palabras clave: aprendizaje, competencias, formación, metodología, evaluación.

\section{Introducción}

En la mayoría de casos, la evaluación condiciona de tal manera la dinámica del aula, donde podría decirse que el objetivo no parece que sea el aprendizaje del alumno sino el superar con éxito el acto de evaluación. De esta forma, la evaluación se convierte en un acto final y supone el fin de proceso de aprendizaje. En el momento en el que el alumno consigue superar el acto de evaluación, se pone un punto y aparte en el proceso de aprendizaje. Es más, podemos decir, que la evaluación no se puede considerar en sí un acto de aprendizaje.(Basterrechea, 2013)

Pero en el momento de la corrección, el profesor puede detectar que el alumno ha superado los conocimientos mínimos en la materia, pero ha demostrado cierta confusión en algunos 
conceptos importantes, pero, pese a ello, en el acto de evaluación, se considera aprobado o superado. En una evaluación tradicional, el alumno pondría punto y aparte, obtendría un aprobado, y en cuanto a estos conceptos que no le quedaban demasiado claros, ya no existirá ningún interés en aclararlos. El objetivo final que se plantea el alumno es la superación del acto de evaluación, y ya se ha conseguido (Castelló \& Cladellas, 2013; Contreras, 2010).

La metodología propuesta en este trabajo consiste en considerar la evaluación como un acto intermedio en el proceso de aprendizaje. El alumno obtiene un feed-back de cuál es su nivel de conocimientos en un momento dado, y tiene la posibilidad de poner en marcha un pensamiento reflexivo acerca de los resultados obtenidos durante el aprendizaje y poner en marcha estrategias para lograr alcanzar mayor nivel de conocimientos que completen su proceso de aprendizaje.(Marques \& Nunes, 2011; Moreno Olivos, 2009)

\section{Metodología de evaluación activa circular.}

Con este método combinado de aprendizaje - evaluación, el alumno realiza una prueba escrita puntuable. Para poner en práctica y evaluar la metodología se ha planteado una prueba tipo test sobre un grupo de 30 alumnos de una asignatura de tercer curso. El método sería la evaluación circular de los contenidos teóricos y conceptuales de una asignatura, pero esta metodología podría ser extrapolable a casos prácticos, problemas, etc. También se podría aplicar sobre pruebas de evaluación que sean específicamente tipo test.

\subsection{Descripción de la prueba.}

El alumno debe estudiar todo el contenido de la asignatura, mediante las herramientas que tiene disponibles. A la clase magistral tradicional y a los apuntes y material facilitado por el profesor, se unen nuevas herramientas multimedia que pueden resultar de gran interés para favorecer el aprendizaje por parte del alumno. En este sentido, destacan herramientas como los videos polimedia, fragmentos de 5 a 10 minutos de duración donde se muestra, de forma sincronizada, la imagen y voz del profesor y una pantalla en la que se muestran los contenidos de una presentación y los screencast, donde se combina la voz del profesor y una pantalla en la que se muestra el funcionamiento generalmente de un programa informático.(Solé, 2009)

Dentro del contenido general habrá unos puntos claves, unos contenidos mínimos imprescindibles para el aprobado de las materias, que el docente deberá marcar como objetivos en el momento de planificar y plantear la asignatura a los alumnos.

En base a estos conocimientos definidos en los objetivos y programa de la asignatura, el docente deberá plantear la prueba escrita. En este caso, con el objetivo de contar con información objetiva para desarrollar el trabajo, se ha decidido elaborar con un test de 30 preguntas, con el que se pueden llegar a abordar los contenidos mínimos de un gran número de asignaturas.

(c)) BY-NC-ND 2016, Universitat Politècnica de València 


\subsubsection{Realización de la evaluación inicial}

En esta primera fase, la evaluación escrita no es distinta a la tradicional. Se trata de realizar un examen test habitual. Es muy importante durante el transcurso de la prueba no permitir hojas en blanco para evitar que los alumnos puedan tener copia escrita de las preguntas de la prueba, así como verificar que todas las hojas de examen sean devueltas al profesor tras la finalización de la prueba.

El tipo de preguntas tipo test cuenta con 4 respuestas posibles. Serán preguntas que tratarán de hacer razonar al alumno con la base de los conocimientos adquiridos, no limitándose a ser preguntas y respuestas que puedan ser memorizadas de una forma mecánica.

Una de las preguntas que podrían servir de ejemplos sería la siguiente:

\section{La densidad del acero es:}
A. $7850 \mathrm{Kg} / \mathrm{m} 3$
B. $7,85 \mathrm{~g} / \mathrm{dm} 3$
C. $0.0785 \mathrm{~g} / \mathrm{mm} 3$
D. Todas las anteriores son correctas.

Se darían 4 respuestas posibles. El alumno debe razonar la respuesta correcta realizando los cambios de unidades de forma correcta. En este caso, la respuesta correcta sería la A.

Esta prueba se puntuará conforme a los criterios habituales de corrección, y se notificará la nota a los alumnos. En este caso se ha utilizado la herramienta desarrollada por la UPV ALCE, para la corrección de pruebas tipo Test.

Los resultados de la prueba realizada como caso de estudio se presentan en la siguiente figura:

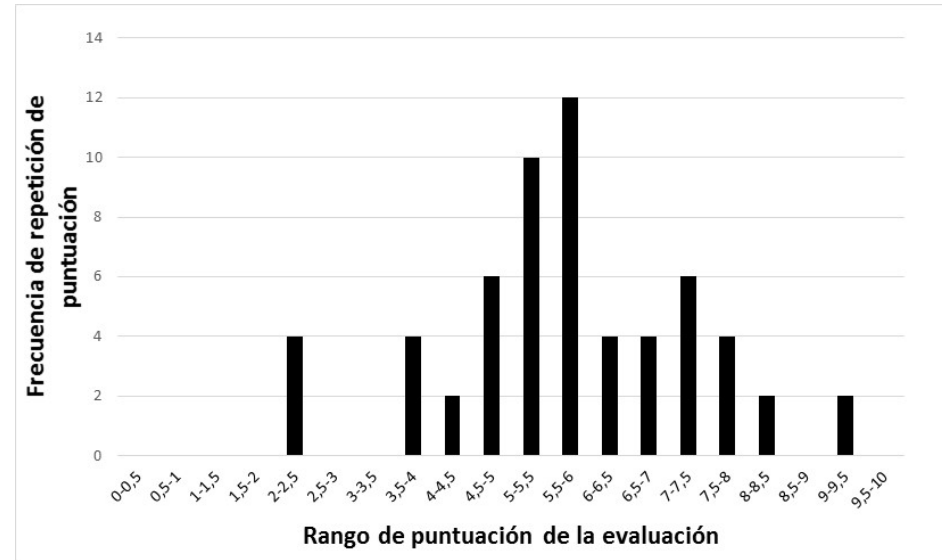

Fig. 1 Tabla de frecuencias de repetición de rangos de nota sobre un grupo de 30 alumnos en el acto de evaluación inicial 


\subsubsection{Realización de la repetición activa de la prueba}

La segunda fase de este proceso de aprendizaje circular es la repetición de la prueba. Destacar que no se trata de una prueba de recuperación. La podemos definir como una fase de reflexión y asimilación de conceptos. Con dos semanas de diferencia, se lleva cabo otra prueba escrita, que denominaremos evaluación circular. La prueba escrita cuenta con el mismo número de preguntas, y además las preguntas son esencialmente las mismas, con matices o pequeñas variaciones, donde se deberá demostrar que efectivamente, el concepto está claro. Al alumno debe ser capaz de identificar los errores cometidos en la primera prueba, y ser capaz de interiorizar los conocimientos de forma que pueda corregir los errores en esta prueba posterior. (Moreno Olivos, 2009).

Si nos remitimos al ejemplo anterior, tendríamos la pregunta test se podría formular de la siguiente forma:

\section{La densidad del acero es:}
A. $78,50 \mathrm{Kg} / \mathrm{m} 3$
B. $7,85 \mathrm{~g} / \mathrm{dm} 3$
C. $0.0785 \mathrm{~g} / \mathrm{mm} 3$
D. Todas las anteriores son incorrectas.

En este caso, la respuesta correcta sería la D. El alumno debería llegar a esta respuesta razonando los conocimientos adquiridos y corrigiendo los posibles errores cometidos en el ejercicio de evaluación primero.

Además, con este tipo de actividad, se fomenta una competencia transversal muy significativa e importante en el proceso de aprendizaje como es el trabajo colaborativo. Este tipo de ejercicio va a provocar que los alumnos formen de forma espontánea pequeños grupos de trabajo, aunque se puede dar el caso (las nuevas tecnologías lo permiten) que se llegue a formar un único gran grupo de trabajo. Se ha detectado una nueva forma de comunicación entre los estudiantes que es la utilización de redes sociales a través de un smartphone creando grupos de comunicación entre los alumnos de una asignatura, donde se crea una conversación participativa entre todos los miembros. Todos ellos podrán participar en esa conversación y aportar ideas, conocimientos, etc. Desde el punto de vista del docente, podría ser muy interesante el participar en el grupo, e incluso ser el administrador del mismo, para comprobar y tener constancia de las aportaciones de los alumnos.(Hamodi, López Pastor, \& López Pastor, 2015; Llargués, Herranz, Sánchez, Calbo, \& Virumbrales, 2015)

Dentro del grupo, y en la siguiente semana de clase los alumnos tratan de forma conjunta de recordar las preguntas del ejercicio, y comentar cuál es la respuesta correcta, y discutir por qué las restantes preguntas no lo son. No basta con recordar la respuesta correcta, sino que habrá que saber razonarla, puesto que en este segundo ejercicio puede cambiar un matiz que haga que la respuesta correcta sea otra.

(cc) EY-NC-ND 2016, Universitat Politècnica de València 


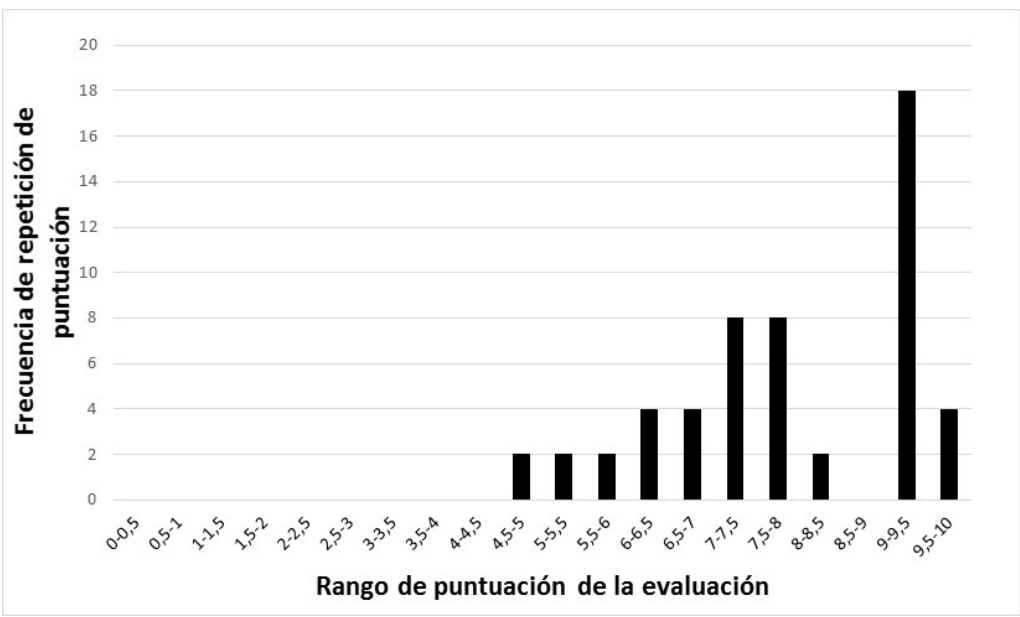

Fig. 2 Tabla de frecuencias de repetición de rangos de nota sobre un grupo de 30 alumnos en el acto de evaluación activa circular

El resultado obtenido, tal como observamos en la Fig.2, ha sido significativamente mejor que el resultado obtenido en la prueba inicial. Esto, en sí, ya es un logro interesante. Sin embargo, en esta segunda prueba únicamente se recompensará la excelencia en el trabajo. Está segunda prueba únicamente tendrá valor para la nota total de la prueba, si se ha alcanzado la excelencia. Es decir, se ha alcanzado como mínimo una nota de 9 en esta segunda prueba. En este caso, se hará un promedio con la nota inicial. En caso contrario, la prueba no tendrá validez alguna en la calificación final de la asignatura.

En la Fig. 3 se puede observar la evolución de la evaluación entre la primera prueba y la prueba activa circular para cada uno de los alumnos. En general, la práctica totalidad de los alumnos han incrementado su puntuación. La excepción la han protagonizado dos alumnos que han cometido más errores en la evaluación activa que en la inicial, muestra inequívoca de su falta de implicación y motivación para el aprendizaje. 


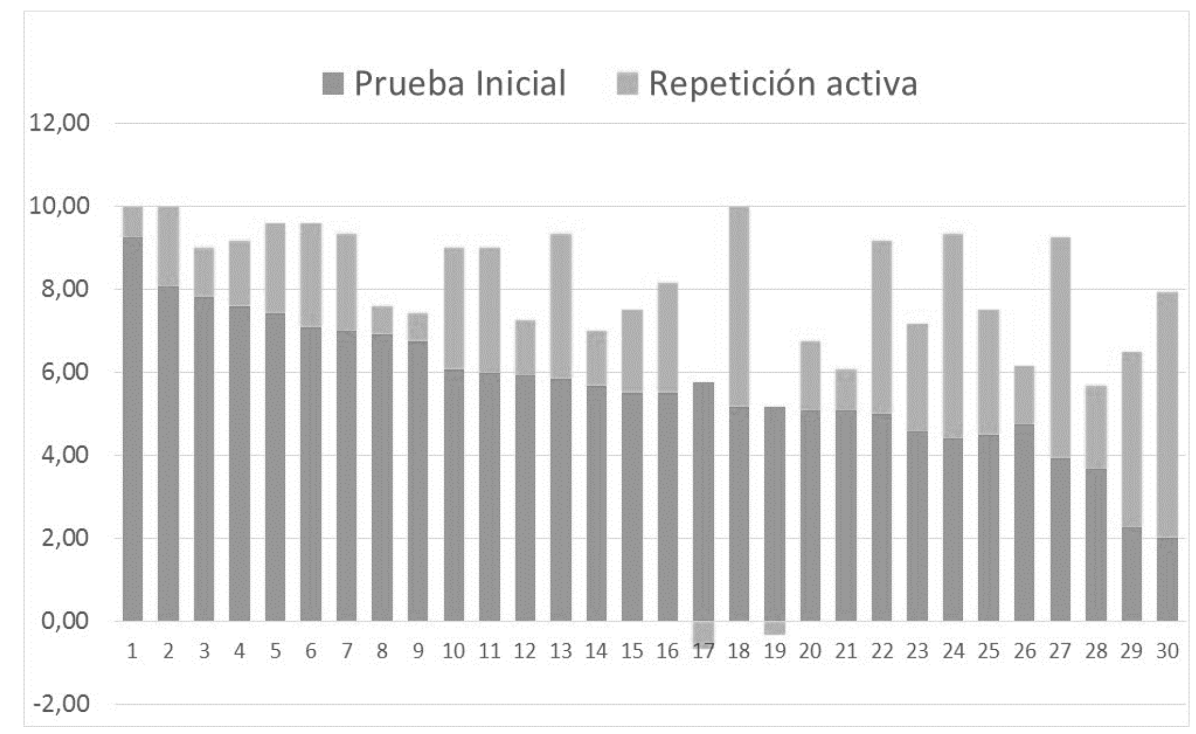

Fig. 3 Evolución de la evaluación entre la prueba inicial y la repetición activa para cada uno de los alumnos.

En vista los resultados obtenidos, podemos establecer el nivel de éxito de la segunda prueba por grupo de alumnos, como vemos en la Tabla 2.

Tabla 2. Resultados de la evaluación activa circular en función de las puntuaciones obtenidas en la prueba inicial

\begin{tabular}{ccc}
\hline $\begin{array}{c}\text { Rango de } \\
\text { puntuaciones } \\
\text { en la prueba } \\
\text { inicial }\end{array}$ & $\begin{array}{c}\text { Alumnos que alcanzan la } \\
\text { nota 9 en la evaluación } \\
\text { circular / Alumnos totales }\end{array}$ & $\begin{array}{c}\text { Porcentaje de } \\
\text { éxito por rango } \\
\text { de puntuaciones }\end{array}$ \\
\hline $0-3$ & $0 / 2$ & $0 \%$ \\
$3-5$ & $2 / 6$ & $33 \%$ \\
$5-7$ & $5 / 15$ & $33 \%$ \\
$7-8$ & $5 / 5$ & $100 \%$ \\
$9-10$ & $2 / 2$ & $100 \%$ \\
\hline
\end{tabular}

Observamos cómo, en general, todos alumnos con buenas puntuaciones alcanzarn el máximo aprendizaje. Con puntuaciones desde 3 a 7 puntos, aproximadamente un tercio de los alumnos son capaces de obtener el máximo aprendizaje, mientras que los alumnos con puntuaciones bajas, son pocos los casos que llegan a alcanzar el máximo nivel de aprendizaje. 


\section{Conclusiones}

Con esta metodología queda demostrado que el nivel de conocimientos general de la asignatura por parte de los alumnos se ha incrementado de forma significativa. La percepción del docente es que los alumnos ven este tipo de repetición activa de la prueba como un reto y una oportunidad, que además cuenta con el factor de la inmediatez. En menos de 2 semanas el alumno tendrá una nueva oportunidad. Y ante esos estímulos, el alumno, en general, ha reaccionado.

Podemos concluir que en general esta metodología resulta positiva para la gran mayoría del grupo. Únicamente dos personas no han superado la puntuación de su prueba inicial, por lo que en general el resultado ha sido satisfactorio en el grupo evaluado. Sería necesario articular algún mecanismo para motivar a que este grupo de alumnos tenga una participación activa. Por otro lado, las conclusiones obtenidas se han basado en un único caso de estudio, ya que se trata de un trabajo iniciado recientemente. Por tanto, las conclusiones pueden tener un cierto sesgo, por lo que sería necesario continuar con esta metodología y establecer conclusiones con mayor soporte de datos de estudio.

\section{Referencias}

Basterrechea, L. (2013). Subjetividad en la didáctica de las carreras proyectuales: Grupos de aprendizaje; evaluación. Cuadernos del Centro de Estudios en Diseño y Comunicación. Ensayos(43), 221-230.

Castelló, A., \& Cladellas, R. (2013). La evaluación de la comprensión en el aprendizaje: El empleo de las TIC en el análisis de estructuras de conocimiento. [The assessment of comprehension in learning: ICT in the knowledge structures' analysis

Avallando a compreensão de aprendizagem: A utilização das TIC na análise de estruturas de conhecimento]. Estudios pedagógicos (Valdivia), 39(Especial), 41-57. doi: 10.4067/s071807052013000300004

Contreras, G. (2010). Diagnóstico de dificultades de la evaluación del aprendizaje en la universidad: un caso particular en Chile. [Diagnosing Learning Assessment Difficulties at the University Level: A Specific Case in Chile

Diagnóstico de dificuldades na avaliação da aprendizagem na universidade: um caso específico em Chile]. Educación y Educadores, 13(2), 219-238.

Hamodi, C., López Pastor, V. M., \& López Pastor, A. T. (2015). Medios, técnicas e instrumentos de evaluación formativa y compartida del aprendizaje en educación superior. [Means, techniques, and instruments of formative and shared assessment of learning in higher education]. Perfiles educativos, 37(147), 146-161.

Llargués, E., Herranz, X., Sánchez, L., Calbo, E., \& Virumbrales, M. (2015). Aplicación de un modelo híbrido de aprendizaje basado en problemas como estrategia de evaluación e interrelación 'multiasignaturas'. [Application of a hybrid model of problem-based learning as a strategy for assessment and the integration of 'multiple subjects']. FEM: Revista de la Fundación Educación Médica, 18(2), 131-137.

Marques, A. S., \& Nunes, L. C. (2011). Possibilidades da participação na avaliação da aprendizagem online no ensino superior. [Possibilities of participation in the evaluation of online learning in higher education

(cc)) EY-NC-ND 2016, Universitat Politècnica de València

Congreso In-Red (2016) 
Posibilidades de participación en la evaluación del aprendizaje online en la enseñanza superior]. Ensaio: Avaliação e Políticas Públicas em Educação, 19(72), 599-622. doi: 10.1590/s010440362011000400008

Moreno Olivos, T. (2009). La evaluación del aprendizaje en la universidad: tensiones, contradicciones y desafíos. Revista mexicana de investigación educativa, 14(41), 563-591.

Solé, M. (2009). Desarrollo de portafolios para el aprendizaje y la evaluación. Educere, 13(45), 544 544. 